\title{
Second Language Learning with Affective Factors and Deep Neural Networks Methods
}

\author{
Meryem Karlik ${ }^{1} \&$ Bekir Karlik ${ }^{2}$ \\ ${ }^{1}$ Tashkent State Transport University, Tashkent, Uzbekistan \\ ${ }^{2}$ McGill University, Montreal, QC, Canada \\ Correspondence: Meryem Karlik, Tashkent State Transport University, Tashkent, Uzbekistan. \\ Email: drmeryemk@gmail.com
}

DOI: $10.53103 /$ cjlls.v1i3.20

\begin{abstract}
The goal of the proposed study is to specify second language learning (SLL) and affective factors among variables of five language skills analyzed by using different Deep Neural Networks methods in freshman class of higher education students. Assessment levels of five language skills of the students have been showed by the percentages and whether there was significant difference between genders or five skills of learners analyzed by statistically by using DNN and FCNN. Questionnaire is evaluated to quantify students' affective factors as knowledge convenient for DNN input. Survey consists of four parts. First part, from 1 to 19 questions, deals with educational background of students with open-ended questions. From second to fourth part, the questionnaire is improved by using Likert's five-level response scale from 1, representing strong agreement to 5, representing strong disagreement. Major factors of these parts are affective factors; motivation, personality and attitude. Second part from 20 to 29 deals with personality, third part from 30 to 47 deals with motivation and last part from 48 to 54 deals with attitude. Data is transferred from survey answers to an excel sheet and converted to the numbers then analyzed with DNN methods. The importance of the study is to highlight right language skill by the help of affective factors for the development of developing second language learning.
\end{abstract}

Keywords: Affective Factors, Deep Neural Networks, Language Skills, Second Language Learning

\section{Introduction}

Teaching foreign language is a very important and difficult task in all over the world. In order to teach effectively, teachers have to know so many things such as attitudes, motivation and knowledge of the students. Teaching quality plays an important role for it can result in successful or unsuccessful learning (Karlik, 2016). Furthermore, most teachers use "one-size-fits-all" approach while teaching (Felder \& Brent 2005). This approach is not appropriate for all because every student has different affective characteristics. According to affective factor, students have different attitudes toward teaching and learning and have different motivation to learn second language. So, with these varieties 
of students, teachers cannot use only one approach because it will not fit for all students. Teachers can utilize a number of ways; select the suitable materials by using different methods while teaching, and determine the most useful teaching materials or sources for the students.

Recently, there are some computer-assisted learning studies in language teaching and learning. Chen, Shih, and Liu (2015) have proposed if and how the instructional design affected the learning outcome in a blog-mediated project from 34 college-level students of English learners from 2 universities in Taiwan. Cardoso (2011) has proposed the results of a research on the use of a learner response system by a group of 30 Brazilians students of English as a foreign language using a methodology that included the analysis of survey questionnaires and open-ended oral interviews. Changa and Hoa (2009) have presented the effects of students' locus of examination and types of examination over instruction on their self-efficacy and performance in a web-based language learning environment. Hung (2015) has proposed the possible impacts of flipping the classroom on English language learners' academic performance, participation levels, and learning attitudes. Tsai et al. (2020) have presented a review paper on deep neural networks with an aim of achieving feasible and interpretable prediction results, thereby improving student learning.

This study presents three different Machine Learning approaches (ANN, DNN and FCNN) in SLL to determine students English learning abilities. The collected dataset about affective factors were analyzed by using different ML methods with major factors; motivation, attitude and personality.

\section{Methodology}

\section{Deep Neural Networks}

A Deep Neural Networks (DNN) aims at learning feature hierarchies with features from higher levels of the hierarchy structured by the composition of lower-level features. It consists of learning techniques for a wide array of deep Multi Layered Perceptron (MLP) structures, including neural networks with more than a hidden layer as defined by Bengio (2009). He recommends that in order to learn the type of complex functions that can indicate high-level abstractions, one may need deep architectures. DNN occurs many interconnected groups of artificial neurons and information of parallel processing using a connectionist approach to calculation. In many cases a DNN is an adaptive system that modifies its structure according to internal or external information that runs through the neural network pending the learning stage. DNN has the computer like capability of increasing the processing power of the brain by information of parallel processing faster.

The basic unit of an ANN is the neuron (or node). Each neuron receives a number of inputs, multiplies the inputs by individual weights, sums the weighted inputs, and transfers the sum up an activation function that can become generally Sigmoid and 
Hyperbolic Tangent Function (Karlik \& Olgac, 2011). The ANN has two stages of operation; the training stage and the testing stage. In the training stage, the neuron can be trained to dismiss (or not), for specific input patterns. In the testing stage, when a taught input pattern is detected at the input, its associated output becomes the network actual output. Commonly ANN are adjusted, or trained, so that a particular input leads to a particular desired output. Typically, many such input/desired output pairs are required for training of ANN (Iseri \& Karlik, 2009). As seen in Fig.1, it is composed of three full connected layers of neurons as input, hidden, and output layers which is called as MultiLayered Perceptron (MLP).

Each neuron receives input from all the neurons in the previous layer and transfers its output to all the neurons in the next layer (Ozbay \& Karlik, 2002). In the ANN learning stage, the weights are updated so that the actual output values are calculated for a range of input values are that as close as possible to the known real values of the sample features. In this study, a three layered and a five-layered MLP architecture were used and trained by using the error Back Propagation (BP) algorithm which is a supervised learning paradigm. $\mathrm{BP}$ algorithm is a mostly used method to analyze complicated data and for classification (Karlik, 2012), optimization (Ayhan, Karlik, \& Tandiroglu, 2004), and prediction (Kizilaslan \& Karlik, 2009). Generally speaking, although BP allows for deep problems in principle, it seemed to work only for shallow problems (Schrauwen, 2007). The Backpropagation training algorithm can be explained as follows:

1. First start by initializing the weights randomly for all neurons given number between 0 and 1 , then:

2. Implement input pattern and propagate the activation function of the network,

3. Compute the error at the output, then back-propagated back to previous layers,

4. Update the weights for each neuron according to the error,

5. Repeat this process till acceptable error value (e.g., error $<1 \%$ ), which means that ANN was trained successfully, or if the network reaches a maximum number of iterations (or nonacceptable error) which means that ANN training was trained unsuccessfully.

The aim of this iteration is to find a good algorithm for adjusting the weights and thresholds in each cycle (step 3) to minimize the error. 


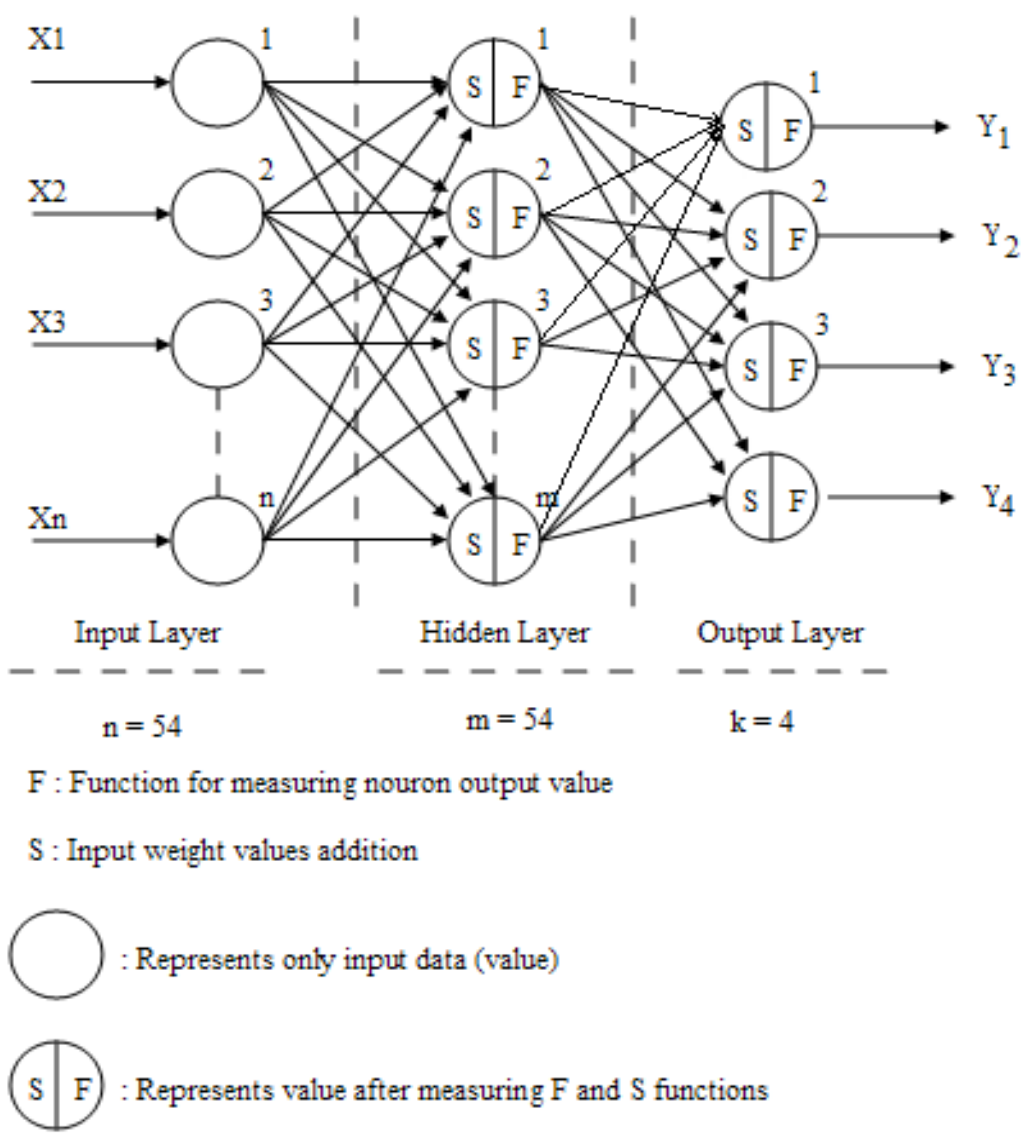

Figure 1: The general MLP architecture of ANN

As seen in Fig. 1, the architecture of MLP for ANN is 54:54:4 that means 54 number of neurons for the input layer, 54 number of neurons for only one hidden layer, and 4 number of neurons for the output layer. Each of the survey answers which was collected from students is applied to inputs in all ML methods.

\section{Fuzzy Clustering Neural Networks}

ANN and DNN are frequently used for classification and show high success in many problems. However, ANNs obtain information, they are not useful because they are not understandable. This poses a problem for many data mining problems where understandable rules are important. For this reason, various methods are developed for removing rules from ANN. Moreover, ANN provides the same performance in a very long training phase in some applications. In this study, an efficient hybrid fuzzy clustering neural network (FCNN) structure has been developed by combining supervised artificial neural networks and unsupervised fuzzy c-mean clustering algorithms as seen in Fig 2. 
There is a cascade connection between them. This hybrid method has been suggested by Karlik et al. $(2002,2003)$ first to solve complicated dataset problems. Later it has been improved by Ceylan et al. $(2011,2015)$ named FCNN type-2. With the developed hybrid structure of cascade connection, it is aimed to increase accuracy of classifier. Recently, Karlik (2015) has demonstrated the superiority of FCNN comparing with several wellknown machine learning algorithms.

All used methods (ANN, DNN and FCNN) primarily consist of 2 steps which are training and test. Student survey answers are used for both training and test. Training data is recorded from 40 students. Test data is recorded from 50 students. Each student has different learning ability such as excellent, very good, good, regular and poor for training and test.

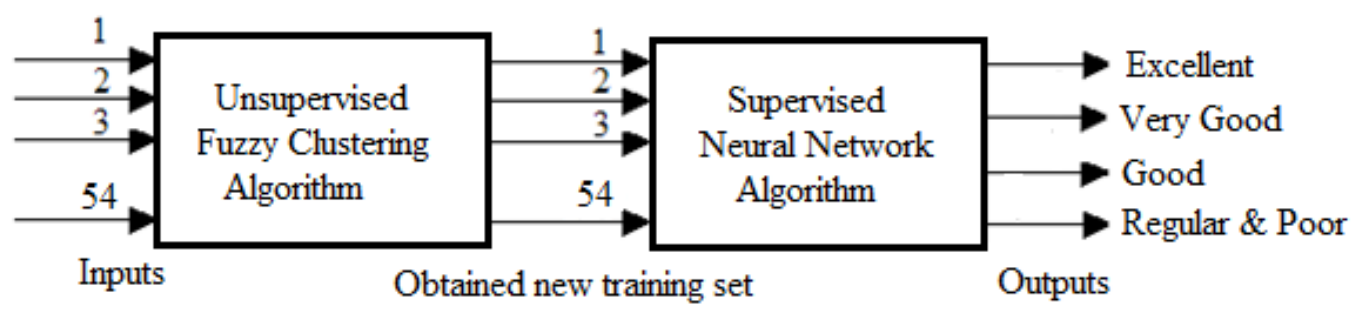

Figure 2: The structure of FCNN algorithm

In this study, Manhattan distance was used. Because, Manhattan distance is more useful if we need to calculate the distance between two data points in a grid-like path like our dataset.

\section{Data Collection and Analysis}

\section{Affective Factors}

Affective factors in second language acquisition investigate variety of issues and play an important role in language learning. These factors are related to learners' emotions and attitudes toward the target language. Schumann (1998) has argued that variable success in second language acquisition is emotionally driven. However, researchers have different opinions about underlying the affective factors. Brown (2007) has argued that affective factors are those factors that come from the learners themselves. According to Krashen's affective filter hypothesis (1998), anxiety is the factor influencing the learning process of students and interacts with other variables like motivation and self-confidence. Generally, researchers classify the factors as; motivation, anxiety, self-esteem, beliefs, prevention, willingness and extro/introversion.

Among these classifications, we propose three essential factors heading the affective factors. These are personality, attitude and motivation. Each of them has 
subdivided factors. The personality factor is categorized as extroversion, introversion, selfesteem, anxiety, and inhibition. The motivation factor is categorized as instrumental, integrative, resultative, global, intrinsic, situational, and task-based. The attitude factor is categorized as the community, English, and learning. In this study, these three major factors are the basis of researching students' success in English learning. The selection of affective factors as main factors depends on the study by Yang \& Damasio (2007) describing the major role of affective factors in learning.

\section{Subjects}

Subjects responding to the survey were 90 students from a foundation university in Bosnia and Herzegovina named International Burch University. The subjects 'major was English. Data were collected from English freshman classes of following departments; Department of Information Technologies (21), Department of Electrical and Electronics Engineering (18), Department of Genetics and Bioengineering (31) from the Faculty of Engineering and Information Technologies, and Department of English Language and Literature (20) from the Faculty of Education.

\section{Data Collection}

Survey is evaluated to quantify students' affective factors as knowledge convenient for ANN input of MLP architecture. Survey consists of four parts. First part from 1 to 19 questions deals with educational background of students with open-ended questions. From second to fourth part, the questionnaire is used using Likert's five-level response scale from 1, representing strong agreement to 5, representing strong disagreement. Major factors of these parts are affective factors; motivation, personality and attitude. Second part from 20 to 29 deals with personality, third part from 30 to 47 deals with motivation and last part from 48 to 54 deals with attitude. This survey is validated by English experts before presenting to the students (Karlik, 2016).

\section{Data Preprocessing}

Firstly, the data is transferred from survey answers to an excel sheet. The dataset has numeric values. But numeric values have different formats. So, they are converted to the numbers which are varying to 0 and 1 which is called as normalized data. Before processing the row data, it should be normalized. One of the well-known normalizations by scaling between 0 and 1 is $\mathrm{min} / \max$ normalization.

Secondly, dataset was divided into training and test data. This dataset consists of 54 attributes. Number of total students are 90 which 40 of them is used for training; the 
others are used for test. Training data consists of $2160(54 \mathrm{x} 40)$ instances. Test data consists of 2700 (54x50) instances (Karlik, 2016).

Both neural networks programs have learned the features with a very low mean squared error (MSE) rate and with 3000 iterations. The iterations are in order to teach better to the program as shown in Fig. 3. This iteration number can be redounded to learn better (Karlik, 2016).

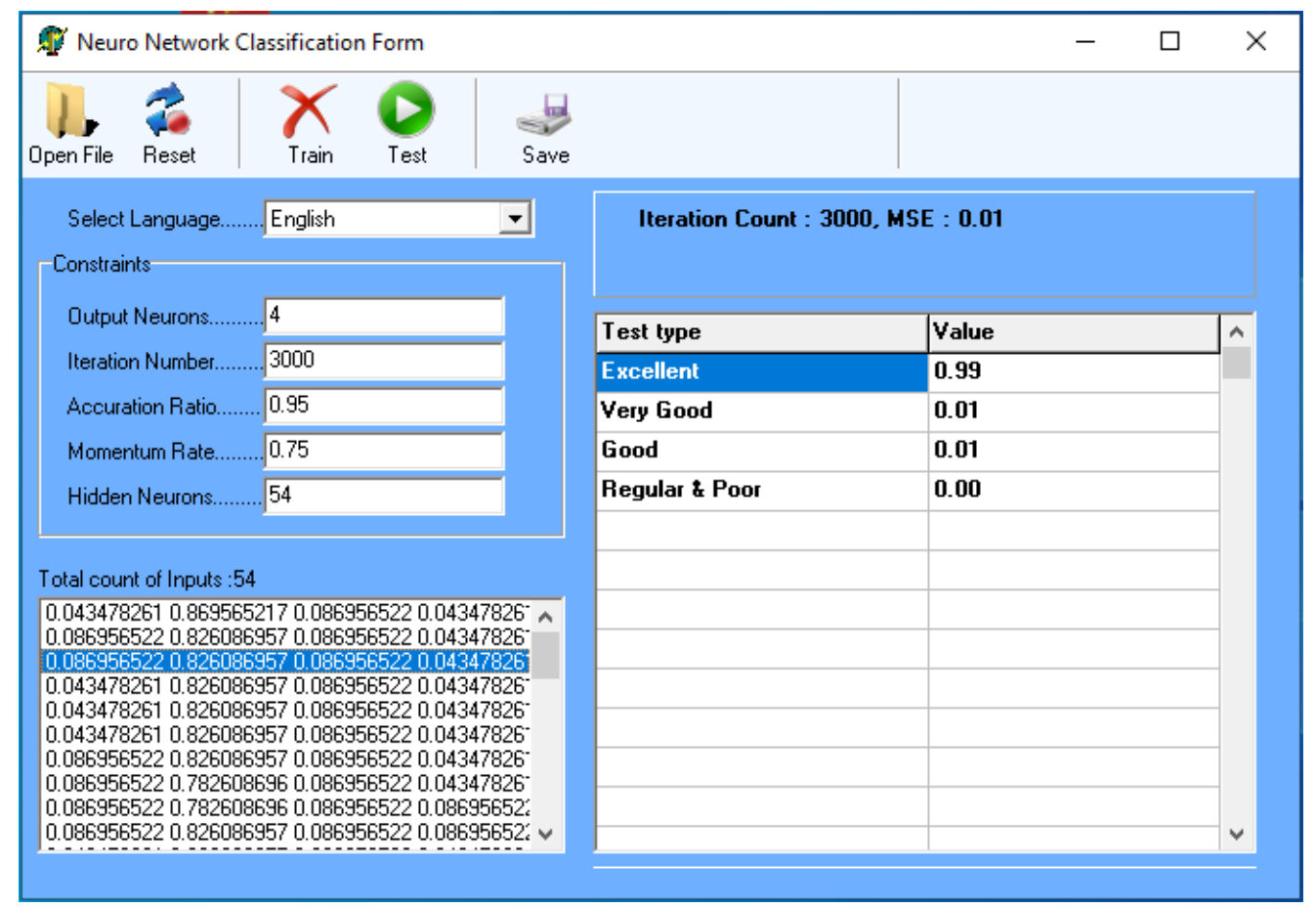

Figure 3: User interface of the ANN

\section{Results and Discussion}

After training data with both ANN and DNN models, test data is used for testing both models. All test results of these models are compared to different abilities as seen from Table 1 to Table 5. In the test results learning ability of general proficiency is given in Table 1. As seen in this table, while 15 students were excellent, 13 accurate estimations identifying as very good instead of 2 excellent for ANN. while 15 students were excellent, 12 accurate estimations were identified as 2 very good and 1 good instead of 3 excellent for DNN. All of them were estimated with FCNN correctly. In the second line, there had to be 13 very good, but there were 10 items that were estimated accurate and 3 of them in excellent were estimated error for both ANN and DNN. While 13 students were very good, 12 accurate estimations were identified as very good instead of only one good for FCNN. 
In the third line, while 15 items in good had to be contemplated, 10 items were generalized accurate, 3 of them in very good and 2 of them in regular \& poor were found error for ANN. while 15 items in good had to contemplate 11 items generalized accurate, 2 of them in excellent and 2 of them in regular \& poor were found error for DNN. While 15 students were very good, 13 accurate estimations were identified as very good instead of 2 excellent for FCNN. All of the students appeared regular \& poor in 7 items were estimated to be accurate for all models.

Table 1: Test results of learning ability of general proficiency

\begin{tabular}{|c|c|c|c|c|c|c|c|c|c|c|c|c|c|c|}
\hline \multicolumn{3}{|c|}{ Excellent } & \multicolumn{3}{|c|}{ Very Good } & \multicolumn{3}{|c|}{ Good } & \multicolumn{3}{|c|}{$\begin{array}{c}\text { Regular \& } \\
\text { Poor }\end{array}$} & \multicolumn{3}{|c|}{ Total } \\
\hline 玄 & 允 & $\sum_{\text {Z }}^{Z}$ & 妾 & 允 & $\sum_{\text {Z }}^{Z}$ & 学 & 允 & $\underbrace{Z}_{\text {Z }}$ & 文 & 文 & $Z_{\text {Z }}^{Z}$ & 芸 & 玄 & $\sum_{\text {Z }}^{Z}$ \\
\hline 13 & 12 & 15 & 2 & 2 & 0 & 0 & 1 & 0 & 0 & 0 & 0 & 15 & 15 & 15 \\
\hline 3 & 2 & 0 & 10 & 10 & 12 & 0 & 1 & 1 & 0 & 0 & 0 & 13 & 13 & 13 \\
\hline 2 & 2 & 2 & 3 & 0 & 0 & 10 & 11 & 13 & 0 & 2 & 0 & 15 & 15 & 15 \\
\hline 0 & 0 & 0 & 0 & 0 & 0 & 0 & 0 & 0 & 7 & 7 & 7 & 7 & 7 & 7 \\
\hline 18 & 16 & 17 & 15 & 12 & 12 & 10 & 13 & 14 & 7 & 9 & 7 & 50 & 50 & 50 \\
\hline
\end{tabular}

In total, while 50 students of 40 were estimated accurate, 10 of them was estimated error for both models. All of these processes were found after 3000 iterations for both proposed neural network models. If we increase the number of iterations, error recognition is reduced. Consequently, estimation error can be calculated as 10/50 $=0.20$ : so, there is $80 \%$ accuracy for both ANN and DNN. We found better accuracy as $47 / 50=94 \%$ by using FCNN. Table 2 shows the test results of all models for listening learning ability. In the first line in this table; while 16 students were excellent 15 of them was accurate estimation, only one of them was identified as very good instead of excellent for ANN. All of them was identified with both DNN and FCNN methods. 
Table 2: Test results of listening learning ability

\begin{tabular}{|c|c|c|c|c|c|c|c|c|c|c|c|c|c|c|}
\hline \multicolumn{3}{|c|}{ Excellent } & \multicolumn{3}{|c|}{ Very Good } & \multicolumn{3}{|c|}{ Good } & \multicolumn{3}{|c|}{$\begin{array}{c}\text { Regular \& } \\
\text { Poor }\end{array}$} & \multicolumn{3}{|c|}{ Total } \\
\hline 玄 & 允 & $\sum_{\text {Z }}^{Z}$ & 文 & 文 & 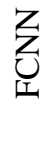 & 㐋 & 允 & 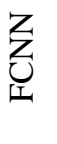 & 学 & 允 & 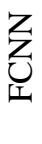 & 㚣 & 䒿 & $\sum_{\text {Z }}^{Z}$ \\
\hline 15 & 16 & 16 & 1 & 0 & 0 & 0 & 0 & 0 & 0 & 0 & 0 & 16 & 16 & 16 \\
\hline 1 & 1 & 1 & 8 & 9 & 9 & 2 & 1 & 1 & 0 & 0 & 0 & 11 & 11 & 11 \\
\hline 0 & 0 & 0 & 2 & 1 & 2 & 14 & 14 & 15 & 0 & 1 & 0 & 16 & 16 & 16 \\
\hline 0 & 0 & 0 & 0 & 0 & 0 & 0 & 1 & 0 & 7 & 6 & 7 & 7 & 7 & 7 \\
\hline 16 & 17 & 17 & 11 & 10 & 10 & 16 & 16 & 16 & 7 & 7 & 7 & 50 & 50 & 50 \\
\hline
\end{tabular}

In the second line; there had to be 11 very good, 8 of them was estimated accurate, one of them excellent and 2 of them in good was estimated error for ANN. For both DNN and FCNN; 9 of them was estimated accurate, one of them excellent and one of them in good was estimated. In third line; while 16 items had to be contemplated in good 14 of them were generalized accurate, and 2 of them in very good were found error for ANN. For DNN; 14 of them were estimated accurate, one of them excellent and one of them in regular \& poor was estimated. While 16 students good, 15 accurate estimations were identified as very good instead of 2 very good for FCNN.

In the last line; while all of the students appeared regular \& poor in 7 items and were estimated to be accurate for both ANN and FCNN, 6 of them was accurate estimation but one of them was identified as good. In total, while 50 students of $15+8+14+7=44$ were estimated accurate, 6 of them was estimated error using ANN, 5 of them was estimated error using DNN. If we increase the number of iterations, error recognition is reduced for both models. Consequently, estimation error for $\mathrm{ANN}=6 / 50=0,12$ : so, there is $88 \%$ success rate. Similarly, estimation error for DNN: $=5 / 50=0,10$ : so, there is $90 \%$ success rate. Finally, estimation error for FCNN: $=4 / 50=0,08$ : so, there is $92 \%$ success rate. 
Table 3: Test results of reading learning ability

\begin{tabular}{|c|c|c|c|c|c|c|c|c|c|c|c|c|c|c|}
\hline \multicolumn{3}{|c|}{ Excellent } & \multicolumn{3}{|c|}{ Very Good } & \multicolumn{3}{|c|}{ Good } & \multicolumn{3}{|c|}{$\begin{array}{l}\text { Regular \& } \\
\text { Poor }\end{array}$} & \multicolumn{3}{|c|}{ Total } \\
\hline 妄 & 允 & $\underbrace{Z}_{\text {Z }}$ & 允 & 允 & $\underset{\text { Z }}{Z}$ & 染 & 荾 & $\underbrace{Z}_{\text {Z }}$ & 岁 & 允 & $\underset{\text { Z }}{Z}$ & 卆 & 㟥 & $\underbrace{Z}_{\text {Z }}$ \\
\hline 15 & 16 & 16 & 1 & 0 & 0 & 0 & 0 & 0 & 0 & 0 & 0 & 16 & 16 & 16 \\
\hline 4 & 2 & 1 & 13 & 15 & 16 & 0 & 0 & 0 & 0 & 0 & 0 & 17 & 17 & 17 \\
\hline 0 & 0 & 0 & 1 & 1 & 0 & 11 & 11 & 12 & 0 & 0 & 0 & 12 & 12 & 12 \\
\hline 0 & 0 & 0 & 0 & 0 & 0 & 0 & 0 & 0 & 5 & 5 & 5 & 5 & 5 & 5 \\
\hline 19 & 18 & 17 & 15 & 16 & 16 & 11 & 11 & 12 & 5 & 5 & 5 & 50 & 50 & 50 \\
\hline
\end{tabular}

In the first line, while 16 students were excellent 15 of them was accurate estimation, only one of them was identified as very good instead of excellent for ANN. All of the students were estimated to be accurate by using both DNN and FCNN. In the second line, there had to be 17 very good, 13 of them were estimated accurate, 4 of them in excellent was estimated error with ANN. With DNN, 15 of them were estimated accurate, 2 of them in excellent were estimated error. While 17 students were very good, 16 accurate estimations were identified as very good instead of only one excellent for FCNN. In the third line, while 12 items had to be contemplated in good 11 of them were generalized accurate, only one in very good were found error. The same result was also found with DNN. However, all of good were defined correctly by FCNN method.

All of the students appeared regular \& poor in 5 items were estimated to be accurate with three models. In total, while 50 students of 44 were estimated accurate, 6 of them were estimated error using ANN. Similarly, 50 students of 47 were estimated accurate, 3 of them were estimated error using DNN. Consequently, estimation error $=6 / 50=0,12$ : so, there is $88 \%$ success rate for ANN. Similarly, estimation error can calculate as $3 / 50=0,06$ : so, there is $94 \%$ success rate for DNN. Finally, estimation error for FCNN: $=1 / 50=0,02$ : so, there is $98 \%$ success rate which showed the best accuracy. 
Table 4: Test results of speaking learning ability

\begin{tabular}{|c|c|c|c|c|c|c|c|c|c|c|c|c|c|c|}
\hline \multicolumn{3}{|c|}{ Excellent } & \multicolumn{3}{|c|}{ Very Good } & \multicolumn{3}{|c|}{ Good } & \multicolumn{3}{|c|}{$\begin{array}{c}\text { Regular \& } \\
\text { Poor }\end{array}$} & \multicolumn{3}{|c|}{ Total } \\
\hline 玄 & 允 & $\sum_{\text {Z }}^{Z}$ & 文 & 允 & $\sum_{\text {Z }}^{Z}$ & 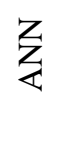 & 允 & $\underbrace{Z}_{\text {Z }}$ & 文 & Z & $\underbrace{Z}_{I}$ & 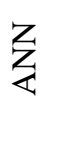 & 允 & $\sum_{\text {Z }}^{Z}$ \\
\hline 11 & 11 & 13 & 2 & 3 & 2 & 2 & 1 & 0 & 0 & 0 & 0 & 15 & 15 & 15 \\
\hline 2 & 1 & 0 & 11 & 12 & 15 & 2 & 2 & 0 & 0 & 0 & 0 & 15 & 15 & 15 \\
\hline 0 & 0 & 0 & 1 & 0 & 0 & 8 & 10 & 9 & 1 & 0 & 1 & 10 & 10 & 10 \\
\hline 0 & 0 & 0 & 0 & 0 & 0 & 0 & 0 & 0 & 10 & 10 & 10 & 10 & 10 & 10 \\
\hline 13 & 12 & 13 & 14 & 15 & 17 & 12 & 13 & 9 & 11 & 10 & 11 & 50 & 50 & 50 \\
\hline
\end{tabular}

In the first line, while 15 students were excellent 11 of them were accurate estimation, 2 of them were identified in very good, other 2 of them were identified in good instead of excellent using ANN. While 15 students were excellent 11 of them were accurate estimation, 3 of them were identified in very good, only 1 of them was identified in good instead of excellent using DNN. Similarly, while 15 students were excellent 13 of them were accurate estimation, 2 of them were identified in very good using FCNN. In the second line, there had to be 15 very good, 11 of them were estimated accurate, 2 of them in excellent and other 2 of them in good were estimated error with ANN. However, for 15 very good, 12 of them were estimated accurate, one of them in excellent and other 2 of them in good were estimated error using DNN. But, all of very good were defined correctly by FCNN method. In the third line, while 10 items had to be contemplated in good 8 of them were generalized accurate, one in very good and one in regular \& poor were found error using ANN. Similarly, while 10 items had to be contemplated in good 9 of them were generalized accurate, only one in regular \& poor were found error using FCNN. However, all of the students appeared good in 10 items and were estimated to be accurate for DNN. Here all of the students appeared regular \& poor in 10 items and were estimated to be accurate for all methods. In total, while 50 students of 40 were estimated accurate, 10 of them were estimated error using ANN. Moreover, 50 students of 43 were estimated accurate, 7 of them was estimated error using DNN. Consequently, estimation error $=10 / 50=0,20$ : so, there is $80 \%$ success rate using ANN. Similarly, estimation error can calculate as $7 / 50=0,14$ : so, there is $86 \%$ success rate for DNN. Finally, estimation error can calculate as $3 / 50=0,06$ : so, there is $94 \%$ success rate for FCNN. 
Table 5: Test results of writing learning ability

\begin{tabular}{|c|c|c|c|c|c|c|c|c|c|c|c|c|c|c|}
\hline \multicolumn{3}{|c|}{ Excellent } & \multicolumn{3}{|c|}{ Very Good } & \multicolumn{3}{|c|}{ Good } & \multicolumn{3}{|c|}{$\begin{array}{l}\text { Regular \& } \\
\text { Poor }\end{array}$} & \multicolumn{3}{|c|}{ Total } \\
\hline 妄 & 允 & $\sum_{\text {Z }}^{Z}$ & 允 & 允 & $\sum_{\text {Z }}^{Z}$ & 玄 & 允 & 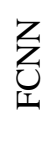 & 允 & 允 & $\underbrace{\text { Z }}_{\text {Z }}$ & 允 & 允 & $\sum_{\text {Z }}^{Z}$ \\
\hline 11 & 10 & 11 & 1 & 0 & 1 & 0 & 0 & 0 & 0 & 2 & 0 & 12 & 12 & 12 \\
\hline 2 & 0 & 1 & 15 & 16 & $\overline{18}$ & 2 & 1 & 0 & 0 & 2 & 0 & 19 & 19 & 19 \\
\hline 0 & 0 & 1 & 2 & 0 & 1 & 6 & 9 & 7 & 2 & 1 & 1 & 10 & 10 & 10 \\
\hline 0 & 0 & 0 & 0 & 0 & 0 & 0 & 0 & 0 & 9 & 9 & 9 & 9 & 9 & 9 \\
\hline 13 & 10 & 13 & 18 & 16 & 20 & 8 & 10 & 7 & 11 & 14 & 10 & 50 & 50 & 50 \\
\hline
\end{tabular}

In the first line, while 12 students were excellent 11 of them were accurate estimation, only one of them was identified as very good instead of excellent using ANN and FCNN. Here, DNN was accurate truly as there were 10 excellent students, the other 2 were estimated regular and poor. In the second line, there had to be 19 very good, 15 of them were estimated accurate, 2 of them in excellent and the other 2 of them in good were estimated error using ANN.

In a similar manner, 16 of them were estimated accurate, 2 of them in regular and poor and one of them in good were estimated error with DNN. However, while 19 students very good, 18 accurate estimations were identified as very good instead of only one excellent for FCNN. In the third line, while 10 items had to be contemplated in good 6 of them were generalized accurate, 2 of them in very good and 2 of them in regular \& poor were found error using ANN. Moreover, only one of them was found error with DNN. All of the students appeared regular \& poor in 9 items and were estimated to be accurate for both methods. In total, while 50 students of 41 were estimated accurate, 9 of them were estimated error using ANN.

Consequently, estimation error $=9 / 50=0,18$ : so, there is $82 \%$ success rate for ANN. Similarly, estimation error can calculate as $6 / 50=0,12$ : so, there is $88 \%$ success rate for DNN. Finally, estimation error can calculate as $5 / 50=0,10$ : so, there is $90 \%$ success rate for FCNN. Apart from these results, we compared four skills according to the gender difference between male and female. For this test we only used conventional ANN which is enough for explaining these skills. The results can be seen from Table 6 to Table 10 .

Here the red ones show incorrect estimates. There are 16 males +34 females $=50$ students. While the error rate in female $=7 / 34=0,205$ and the success rate in female $=27 / 34$ $=79,41 \%$, the error rate in male $=3 / 16=0,1875$ and the success rate in male $=13 / 16=$ $81,25 \%$.

Briefly, the success rates of male and female students are almost the same. Both genders have different skills or affective factors to determine their ability and to learn the 
language. While in writing and listening, females are more successful than males, in reading and speaking it is vice versa. General proficiency balances the success of the genders level.

Table 6: General Proficiency learning ability according to the gender

\begin{tabular}{|c|c|c|c|c|c|c|c|c|c|c|c|c|}
\hline 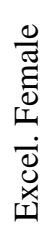 & 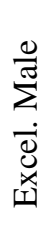 & 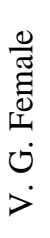 & 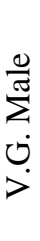 & 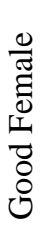 & $\begin{array}{l}\frac{0}{\pi} \\
\frac{\pi}{8} \\
0 \\
0\end{array}$ & 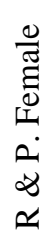 & 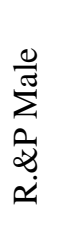 & 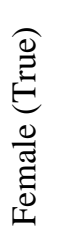 & 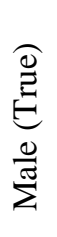 & 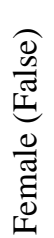 & 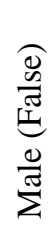 & 胥 \\
\hline 6 & 7 & 1 & 0 & 1 & 0 & 0 & 0 & 7 & 6 & 1 & 1 & 15 \\
\hline 1 & 1 & 9 & 1 & 1 & 0 & 0 & 0 & 9 & 1 & 2 & 1 & 13 \\
\hline 0 & 0 & 3 & 0 & 6 & 4 & 1 & 1 & 6 & 4 & 4 & 1 & 15 \\
\hline 0 & 0 & 0 & 0 & 0 & 0 & 5 & 2 & 5 & 2 & 0 & 0 & 7 \\
\hline 7 & 8 & 13 & 1 & 8 & 4 & 6 & 3 & 27 & 13 & 7 & 3 & 50 \\
\hline
\end{tabular}

Table 7: Listening learning ability according to the gender

\begin{tabular}{|c|c|c|c|c|c|c|c|c|c|c|c|c|}
\hline 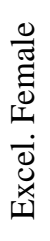 & 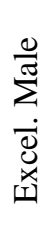 & 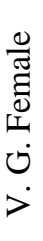 & $\begin{array}{l}\frac{0}{J} \\
\sum_{0}^{J} \\
\dot{>}\end{array}$ & 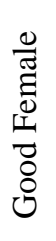 & $\begin{array}{l}\frac{0}{\pi} \\
\sum_{0}^{\pi} \\
0 \\
0\end{array}$ & 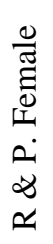 & $\begin{array}{l}\frac{0}{\pi} \\
\sum^{\pi} \\
\frac{\alpha}{\infty} \\
\dot{\alpha}\end{array}$ & 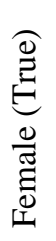 & 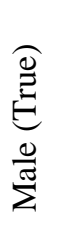 & 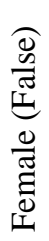 & 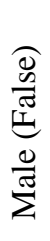 & 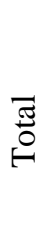 \\
\hline 7 & 8 & 0 & 1 & 0 & 0 & 0 & 0 & 7 & 8 & 0 & 1 & 16 \\
\hline 0 & 1 & 2 & 7 & 1 & 0 & 0 & 0 & 2 & 7 & 1 & 1 & 11 \\
\hline 0 & 0 & 1 & 1 & 11 & 3 & 0 & 0 & 11 & 3 & 1 & 1 & 16 \\
\hline 0 & 0 & 0 & 0 & 0 & 0 & 2 & 5 & 2 & 5 & 0 & 0 & 7 \\
\hline 7 & 9 & 3 & 9 & 12 & 3 & 2 & 5 & 22 & 23 & 2 & 3 & 50 \\
\hline
\end{tabular}

There are 16 males +34 females $=50$ students. While the error rate in female $=2 / 24$ $=0,0833$ and the success rate in female $=22 / 24=91,66 \%$, the error rate in male $=3 / 26=$ 0,115 and the success rate in male $=23 / 26=88,46 \%$. In listening, female students are about $3 \%$ more successful than male students. This can be explained by the nature of the gender. For example, when females are listening someone or something, they listen 
seriously with total attention not to miss any point. And also, they can comment or use some mimics to show that the attention is given for any detail. While males are listening, they keep silent not responding or commenting anything and they do not give full attention 2 so they can miss details in the conversation (Karlik, 2016).

There are 19 males +31 females $=50$ students. While the error rate in female $=4 / 31$ $=0,129$ and the success rate in female $=27 / 31=87,1 \%$, the error rate in male $=2 / 19=$ 0,105 and the success rate in male $=17 / 19=89,47 \%$. In reading, male students are a little more successful than female students. There is not much difference between genders. Both of them have almost the same level of understanding reading. Bacon (1992) found that "men reported using translation strategies more than women while the women reported monitoring their comprehension more."

Table 8: Reading learning ability according to the gender

\begin{tabular}{|c|c|c|c|c|c|c|c|c|c|c|c|c|}
\hline 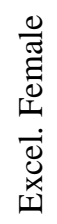 & 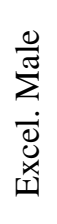 & 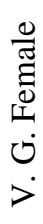 & $\begin{array}{l}\frac{0}{\pi} \\
\dot{\Sigma} \\
\dot{\nu}\end{array}$ & 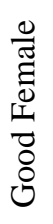 & $\begin{array}{l}\frac{0}{\pi} \\
\sum_{0}^{\circ} \\
\overline{8} \\
0\end{array}$ & 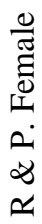 & $\begin{array}{l}\frac{0}{\pi} \\
\sum_{\infty}^{J} \\
\dot{\alpha} \\
\dot{\alpha}\end{array}$ & 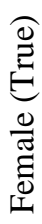 & 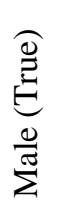 & 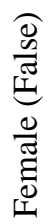 & 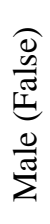 & 吾 \\
\hline 7 & 8 & 1 & 0 & 0 & 0 & 0 & 0 & 7 & 8 & 1 & 0 & 16 \\
\hline 3 & 1 & 9 & 4 & 0 & 0 & $\overline{0}$ & $\overline{0}$ & 9 & 4 & 3 & 1 & 17 \\
\hline 0 & 0 & 0 & 1 & 9 & 2 & 0 & 0 & 9 & 2 & 0 & 1 & 12 \\
\hline 0 & 0 & $\overline{0}$ & 0 & 0 & 0 & 2 & 3 & 2 & 3 & $\overline{0}$ & 0 & 5 \\
\hline 7 & 9 & 3 & 9 & 12 & 3 & 2 & 5 & 27 & 17 & 4 & 2 & 50 \\
\hline
\end{tabular}

There are 15 males +35 females $=50$ students. While the error rate in female $=7 / 35$ $=0,2$ and the success rate in female $=28 / 35=80 \%$, the error rate in male $=3 / 15=0,2$ and the success rate in male $=12 / 15=80 \%$. 
Table 9: Speaking learning ability according to the gender

\begin{tabular}{|c|c|c|c|c|c|c|c|c|c|c|c|c|}
\hline 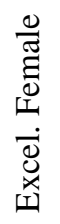 & $\begin{array}{l}\frac{0}{\tilde{J}} \\
\sum^{\pi} \\
\dot{0} \\
\text { x } \\
\text { II }\end{array}$ & 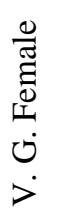 & 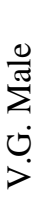 & 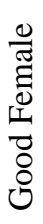 & 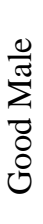 & 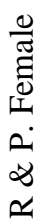 & 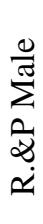 & 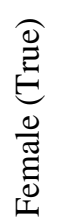 & 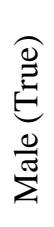 & 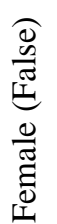 & 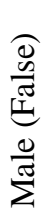 & $\begin{array}{c}\bar{\sigma} \\
\stackrel{0}{0} \\
\ominus\end{array}$ \\
\hline 6 & 5 & 2 & 2 & 0 & 0 & 0 & $\overline{0}$ & 6 & 5 & 2 & 2 & 15 \\
\hline 2 & 0 & 8 & 3 & 2 & 0 & 0 & 0 & 8 & 3 & 4 & 0 & 15 \\
\hline 0 & 0 & 0 & 1 & 8 & 0 & 1 & $\overline{0}$ & 8 & 0 & 1 & 1 & 10 \\
\hline 0 & 0 & 0 & $\overline{0}$ & 0 & 0 & 6 & 4 & 6 & 4 & 0 & 0 & 10 \\
\hline 8 & 5 & 10 & 6 & 10 & 3 & 7 & 4 & 28 & 12 & 7 & 3 & 50 \\
\hline
\end{tabular}

In speaking, the success rates of male and female students are the same. Here both females and males have different but effective motivation towards speaking foreign language. Females interested in foreign language are integrative to manifest that they like the culture and the country. Whereas males' reasons to learn language is mainly instrumental for communicate foreign girls or in order to socialize in the community.

Table 10: Writing learning ability according to the gender

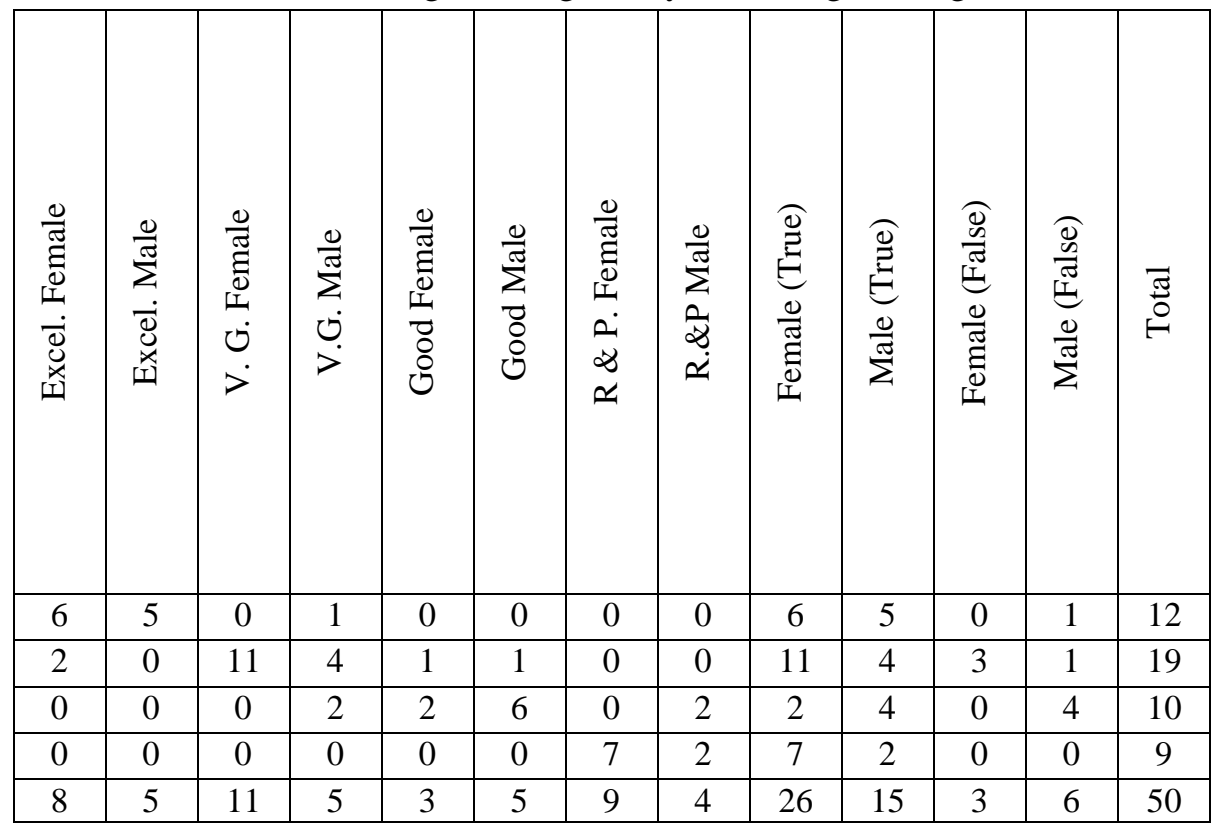

There are 21 males +29 females $=50$ students. While the error rate in female $=3 / 29$ 
$=0,1034$ and the success rate in female $=26 / 29=89,65 \%$, the error rate in male $=6 / 21=$ 0,2857 and the success rate in male $=15 / 21=71,43 \%$. In writing, female students are much more successful than male students. Here we can say that females' motivation towards the language is more confident because they believe that the language will be useful for their future career for norms of the cultures, they are less talkative so they want to express themselves in writing.

\section{Conclusion}

This study has presented English learning abilities of students according to the affective factors by using ANN, DNN and FCNN. The students' ability and gender difference with both methods and the results of individual scores were identified as;

- general proficiency $80 \%$, writing $82 \%$, reading $88 \%$, speaking $80 \%$, listening $88 \%$ by using ANN.

- general proficiency $80 \%$, writing $88 \%$, reading $94 \%$, speaking $86 \%$, listening $90 \%$ by using DNN.

- general proficiency $94 \%$, writing $90 \%$, reading $98 \%$, speaking $94 \%$, listening $92 \%$ by using FCNN.

Averages of ANN, DNN and FCNN were found 83,6\%, 86,8\%, and 93,6\% respectively. According to these results, DNN is naturally better than conventional ANN to make estimation of students learning abilities and gender difference with affective factors. It should be taken into consideration that there is a long training time due to the excess number of layers and weights of DNN architecture. However, hybrid FCNN algorithm provided the best accuracy for all affective factors. DNN algorithm can show good accuracy for big data applications. But hybrid methods are better performance in data with many features (Karlik, 2016; De Campos Souza, 2020). Moreover, we identify individual gender difference by both methods and the results of individual scores using only ANN as follow:

- The results of males are; general proficiency $81,25 \%$, writing $71,43 \%$, reading $89,47 \%$, speaking $80 \%$, listening $88,46 \%$.

- The results of females are; general proficiency $79,41 \%$, writing $89,65 \%$, reading $87,1 \%$, speaking $80 \%$, listening $91,66 \%$.

It is not necessary to use the similar test for DNN and FCNN. Because the results are similar for genders except accuracies.

In the future works, we are planning to use this software to solve the other learning factors. Moreover, the other hybrid or deep learning algorithms can also use to solve similar problems. 


\section{References}

Ayhan, T., Karlik, B., \& Tandiroglu, A. (2004). Flow geometry optimization of channels with baffels using neural networks and second-law of thermodynamics. Computational Mechanics, 33(2), 139-143.

Bengio, Y. (2009). Learning deep architectures for AI. Foundations and Trends in Machine Learning, 2, 1-127. Also published as a book. Now publishers. 2009.

Bacon, S. (1992). The relationship between gender, comprehension, processing strategies, and cognitive and affective response in second-language listening. Modern Language Journal, 76, 160-178.

Brown, D. H. (2007). Principles of language learning and teaching. New York: Pearson Education Inc.

Cardoso, W. (2011). Learning a foreign language with a learner response system: The students' perspective. Computer Assisted Language Learning, 24(5), 393-417.

Ceylan, R., Ozbay, Y., \& Karlik, B. (2011). Integration of type-2 fuzzy clustering and wavelet transform in a neural network-based ECG classifier. Expert Systems with Applications, 38, 1004-1010.

Ceylan, R., Ozbay, Y., \& Karlik, B. (2014). Comparison of type-2 fuzzy clusteringbased cascade classifier models for ECG arrhythmias. Biomedical Engineering: Applications, Basis and Communications (BME), 26(6), 2014, 1450075.

Changa, M. M., \& Hoa, C. M. (2009). Effects of locus of control and learner-control on web-based language learning. Computer Assisted Language Learning, 22(3), 189-206.

Chen, W. C., Shih Y. C. D., \& Liu, G. (2015). Task design and its induced learning effects in a cross-institutional blog-mediated telecollaboration. Computer Assisted Language Learning, 28(4), 285-305.

De Campos Souza, P.V. (2020). Fuzzy neural networks and neuro-fuzzy networks: A review the main techniques and applications used in the literature. Applied Soft Computing, 92, 106275.

Felder, R., \& Brent, R. (2005). Understanding student differences. Journal of Engineering Education, 94(1), 57-72.

Hung, H. T. (2015). Flipping the classroom for English language learners to foster active learning. Computer Assisted Language Learning, 28(1), 81-96.

Iseri, A., \& Karlik, B. (2009). An artificial neural networks approach on automobilepricing. Expert Systems with Applications, 36:2, part. 1, 2155-2160.

Karlik, B., \& Olgac, A.V. (2011). Performance analysis of various activation functions in generalized MLP architectures of neural networks. International Journal of Artificial Intelligence and Expert Systems, 1(4), 111-122.

Karlik, B. (1999). Differentiating type of muscle movement via AR modeling and neural networks classification of the EMG. Turkish J Elec Engin,. 7(1-3), 45-52.

Karlık, B., Tokhi, O., \& Alc1, M. A. (2002). Novel technique for classification of myoelectric signals for prosthesis, 15th Triennial World Congress, (IFAC'02), Barcelona, Spain.

Karlık, B., Tokhi, O., \& Alc1, M. (2003). A fuzzy clustering neural network 
architecture for multi-function upper-limb prosthesis. IEEE Trans on Biomedical Eng. 50(11), 1255-1261.

Karlık, B. (2016). The positive effects of fuzzy c-mean clustering on supervised learning classifiers. Inter. Journal of Artificial Intelligence and Expert Systems, $7(1), 1-8$.

Karlik, M., \& Akbarov, A. (2016). Investigating the effects of personality on second language learning through artificial neural networks. International Journal of Artificial Intelligence and Expert Systems (IJAE). 7(2), 25-36.

Karlik, M. (2016). Artificial intelligence approach in second language learning: Students' English learning abilities. International Burch University, Bosnia and Herzegovina, p.164. Ref.No. 02-01-09-III/16.

Karlik, M. (2017). Artificial neural networks methodology for second language learning. LAP Lambert Academic Publishing, Germany.

Kizilaslan, R., \& Karlik, B. (2009). Combination neural networks forecasters for monthly natural gas consumption prediction. Neural Network World, 19:2, 191199.

Krashen, S. (1998). Second language acquisition and second language learning, London: Prentice Hall International (UK).

Ozbay, Y., \& Karlik, B. (2002). A fast training back-propagation algorithm on windows. Proc. of the Third Inter. Symposium on Mathematical \& Computational Applications, 204-210.

Schumann, J. H. (1998). The neurobiology of affect in language. Language Learning, 48: Supplement 1, ix-326.

Schrauwen, B., Verstraeten, D., \& Van Campenhout, J. (2007). An overview of reservoir computing: theory, applications and implementations. Proc of the 15th European Symposium on Artificial Neural Networks, 471-482.

Tsai, S.C., Chen, C.H., \& Shiao, Y.T. (2020). Precision education with statistical learning and deep learning: a case study in Taiwan. Int J Educ Technol High Educ. 17, 12.

Yang, M. I., \& Damasio, A. (2007). We feel, therefore we learn: The relevance of affective and social neuroscience to education. Mind, Brain, and Education, 1(1), 3-10. 